\title{
Climatic features of the Mediterranean Sea detected by the analysis of the longwave radiative bulk formulae
}

\author{
M. E. Schiano ${ }^{1}$, M. Borghini ${ }^{1}$, S. Castellari ${ }^{1,3}$, C. Luttazzi $^{2}$ \\ ${ }^{1}$ IOF-CNR, Forte S. Teresa, Pozzuolo di Lerici (SP), Italy \\ ${ }^{2}$ IFA-CNR, Rome, Italy \\ ${ }^{3}$ Istituto Nazionale di geofisica, Rome, Italy
}

Received: 25 February 2000 / Revised: 19 July 2000 / Accepted: 25 July 2000

\begin{abstract}
Some important climatic features of the Mediterranean Sea stand out from an analysis of the systematic discrepancies between direct measurements of longwave radiation budget and predictions obtained by the most widely used bulk formulae. In particular, under clear-sky conditions the results show that the surface values of both air temperature and humidity over the Mediterranean Sea are larger than those expected over an open ocean with the same amount of net longwave radiation. Furthermore, the twofold climatic regime of the Mediterranean region strongly affects the downwelling clear-sky radiation. This study suggests that a single bulk formula with constant numerical coefficients is unable to reproduce the fluxes at the surface for all the seasons.
\end{abstract}

Key words: Meteorology and Atmospheric dynamics (radiative processes) - Oceanography: general (marginal and semienclosed seas; marine meteorology)

\section{Introduction}

An important component of the Earth's climate system is the net radiation budget at the ocean surface. Due to the scarcity of direct radiation measurements, in many applications the radiative fluxes at the sea surface are achieved by means of bulk formulae. These are empirical equations giving the radiative energy fluxes in terms of routine ship meteorological observations. Different formulae have been proposed and employed over the years, but their reliability is still questioned. In fact, although the main physical relationships governing the energy exchange processes are not completely neglected, the functional forms and the numerical coefficients of

Correspondence to: M. E. Schiano

e-mail: schiano@iof.cnr.it these equations have been empirically obtained by regression fittings on experimental data sets. Therefore, the bulk formulae are strongly dependent on the observations from which they have been derived and consequently it is not surprising that significant differences are found between predictions and measurements. Nevertheless, the Mediterranean Sea (Med) is a particular case. In fact, all tests that have been conducted to assess the applicability of radiative bulk formulae over the Med by comparing estimates with direct observations show a quasi-systematic overestimation of the downward radiation.

Bignami et al. (1995) compared net longwave fluxes calculated by eight bulk formulae with direct measurements collected during seven cruises in the western Mediterranean (WMed). They found a systematic underestimation (about $30 \mathrm{~W} \mathrm{~m}^{-2}$ ) of the values from the used formulae compared with those from the measurements, except for the Hanstenrath and Lamb (1978) formula. This formula gave estimates above the measurements, but qualitatively in disagreement with them. Bignami et al. (1995) ascribed the failure of the other seven formulae to an overestimation of the downward component of the net flux. Consequently, they developed a new empirical formula to be used for computing the net longwave flux over the Med. Their formula is now used in many studies dealing with the heat budget of the Med. The use of this formula, rather than other ones, indirectly confirms that there is a need to reduce the incident longwave radiation over the Med. Finally, Schiano (1996) compared mean daily solar radiation values (obtained by direct measurements during ten cruises in the Med) to estimates by the Reed (1977) formula. Schiano (1996) found that this formula overestimated the clear-sky insolation and proposed a correction in addition to the one suggested by Gilman and Garrett (1994). She adjusted the numerical coefficients in the formula for the clear-sky radiation according to the amount of surface water vapour.

These previous studies have shown that the most widely used radiative bulk formulae are unable to correctly represent the relationship between the surface 
meteorological parameters and the surface radiative fluxes in the Med. In particular, a systematic overestimation of the short and longwave incident radiation has been shown.

The goal of this study is to use the systematic discrepancies between the radiative fluxes observations and the bulk formulae estimates over the Med to detect some important climate characteristics of this particular area. The approach used to perform this analysis is described in the next section, while the results and discussion are presented in the Results section followed by some final remarks.

\section{Data and analysis procedure}

The quasi-systematic overestimation of the downward fluxes suggests that the failure of the bulk formulae is due to the peculiarities of the Med, rather than the coarseness of the formulae in parametrising the physics of the radiative processes in the atmosphere.

Thus, a detailed analysis of the discrepancies may be used to evidence some features of this area. In order to achieve this goal we decided to use an inverse approach. The radiative bulk formula successfully tested in a midlatitude region is assumed as reference and the discrepancy between predictions and observations is assumed to be due to peculiarities of the surface input parameters rather than to the formula used. We have tried to estimate the values of the surface parameters, which allow the best fit between the predictions and the observations, by inverting the formula. Hence, by comparing the estimated values and the observed ones, we are able to detect some features of the Mediterranean marine environment responsible of the observed discrepancies. This may also suggest how the bulk formulae need to be adjusted for their usage in the Med.

The study is principally achieved for the longwave term of the radiative budget. The net longwave radiation is a powerful climatic index, since it is related to the main important parameters determining the climate of the area under study, like air and sea temperature, humidity and cloud cover.
We have decided to carry out the analysis only for clear-sky conditions, because large errors are due to the usage of the total cloud cover index in the net longwave formulae. We selected the formula of Clark et al. (1974) to perform the analysis. Different tests made over the open ocean (Katsaros, 1990; Josey et al., 1997) show that this formula performs well and it may be used in climatological studies at mid-latitudes. The analysis is conducted by using 838 hourly measurements of atmospheric radiation, sea surface temperature and surface meteorological parameters collected under clear-sky conditions. The data have been obtained during 13 cruises in all seasons in the whole WMed (Table 1). The downward longwave radiation was measured by two Eppley precision infrared radiometers (PIR) mounted on the upper deck of the vessel at about $15 \mathrm{~m}$ above sea level. The instruments locations were carefully selected to minimise the influence of the ship's structure, that is, by avoiding having the two sensors simultaneously in the shadow of the ship's superstructure. The instrumental calibration was checked before and after each cruise. Calibrations were performed in our laboratory using a blackbody cavity and/or by the Eppley calibration service. Accurate measurements of the longwave flux are difficult to make. According to Fairall et al. (1998) we must assign an accuracy of about $5 \%$ to our measurements, since we logged only the battery compensated thermopile outputs. However, we feel that our accuracy is higher. In fact, ignoring the dome effects causes an error. Nevertheless, most of our measurements were taken while the ship was moving and the forced ventilation reduces the effects due to the dome heating. This heating, in any case, introduces a positive bias in the longwave flux, i.e. the observed overestimation of the incident radiation should be reinforced. In addition, we made a separate analysis of daytime and nighttime data and we obtained the same results. Finally, the use of a couple of sensors and the analytic method developed for checking the data quality (Schiano et al., 1993) should increase the accuracy of the longwave radiation data.

The sea surface temperature was measured by using infrared thermometers (PRT-5, Minirad-RS20, Everest
Table 1. Period, working area and number of clear-sky observations of the 13 cruises

\begin{tabular}{llc}
\hline Period & Working area & $\begin{array}{c}\text { Number of } \\
\text { observations }\end{array}$ \\
\hline September 28 to October 7, 1989 & North Tyrrhenian Sea & 20 \\
February 17-22, 1990 & North Tyrrhenian Sea & 35 \\
November 10-19, 1990 & Ligurian Sea & 73 \\
April 20 to May 5, 1991 & Ligurian Sea & 13 \\
October 24 to November 8, 1991 & South Tyrrhenian Sea & 32 \\
April 21 to May 5, 1992 & South Tyrrhenian Sea & 89 \\
August 12-30, 1992 & Balearic Sea & 136 \\
February 27 to March 15, 1993 & South Tyrrhenian Sea & 18 \\
November 4-22, 1993 & Sicilian Channel & 15 \\
May 17-29, 1994 & Sicilian Channel & 67 \\
October 10-20, 1994 & Sicilian Channel & 13 \\
July 4-30, 1995 & Western Mediteranean Sea and & 310 \\
January 10-30,1997 & Sicilian Channel & 17
\end{tabular}


112 ALCS, Tasco THI-500). Measurements of the principal meteorological parameters (air temperature, humidity and air pressure) were obtained by an automatic standard meteorological station mounted onboard and by hourly manual observations. All automatic signals were continuously recorded on the same PC with a sampling rate of $1 \mathrm{~Hz}$. Humidity and air temperature data were checked against an Assmann phychrometer that was used to make hourly manual measurements. A detailed description of the instrumentation used, calibration procedures and data analysis is given by Schiano et al. (1993).

\section{Results}

\section{Peculiarities of the surface parameters}

Following Josey et al. (1997), the downward clear-sky radiation can be obtained by the formula of Clark et al. (1974):

$I R_{n}=\varepsilon \sigma T_{s}^{4}(0.39-0.05 \sqrt{e})+4 \varepsilon \sigma T_{s}^{3}\left(T_{s}-T_{a}\right)$

as:

$I R_{d}=\left(\varepsilon \sigma T_{s}^{4}-I R_{n}\right) /(1-\eta)$

where: $I R_{n}$ is the net longwave radiation $\left(\mathrm{W} \mathrm{m}^{-2}\right), I R_{d}$ is the downward longwave radiation $\left(\mathrm{W} \mathrm{m}^{-2}\right), T_{s}$ is the sea surface temperature $(\mathrm{K}), T_{a}$ is the surface air temperature $(\mathrm{K}), e$ is the surface water vapour tension $(\mathrm{mb}), \varepsilon$ is the water emissivity (0.98), $\eta$ is the longwave reflectance (0.045), and $\sigma$ is the Stefan-Bolzmann constant (5.6697 $10^{-8} \mathrm{~W} \mathrm{~m}^{-2}$ ).

The water vapour tension is the first parameter to be examined, since many authors indicate the parametrisation of this term as the main source of error.

Equation (1) is solved for the term $e$ by using the experimental values of all the other parameters. The mean difference between measured and computed values is about $7.4 \mathrm{mb}$. This result may indicate that the large amount of water vapour in the lower atmosphere could be responsible of the failure of the formula, but the difference in water vapour is too large to be acceptable. In fact, given a value of downward radiation, the corresponding values of the measured water vapour extend over a more limited range (Fig. 1a).

On the other hand, the surface water vapour tension depends on the surface air temperature and the humidity $(U)$ through well-known thermodynamic relationships. Keeping unchanged the values of $T_{a}$, the water vapour tension values obtained are possible only with very low values of $U$ (less than $40 \%$ ), which are not typical of this area. Hence, we argue that it is necessary to consider the $T_{a}$ and $e$ together. We have kept unchanged the measured $U$ and we have derived the corresponding values of $T_{a}$ and $e$, which allow the agreement between predictions and measurements of $I R_{d}$. Both air temperature and water vapour tension must be lower to satisfy Eq. (1). The difference between measured and computed values is not a constant bias. On average, it is about $2.4 \mathrm{mb}$ for water vapour tension and $2.5 \mathrm{~K}$ for air
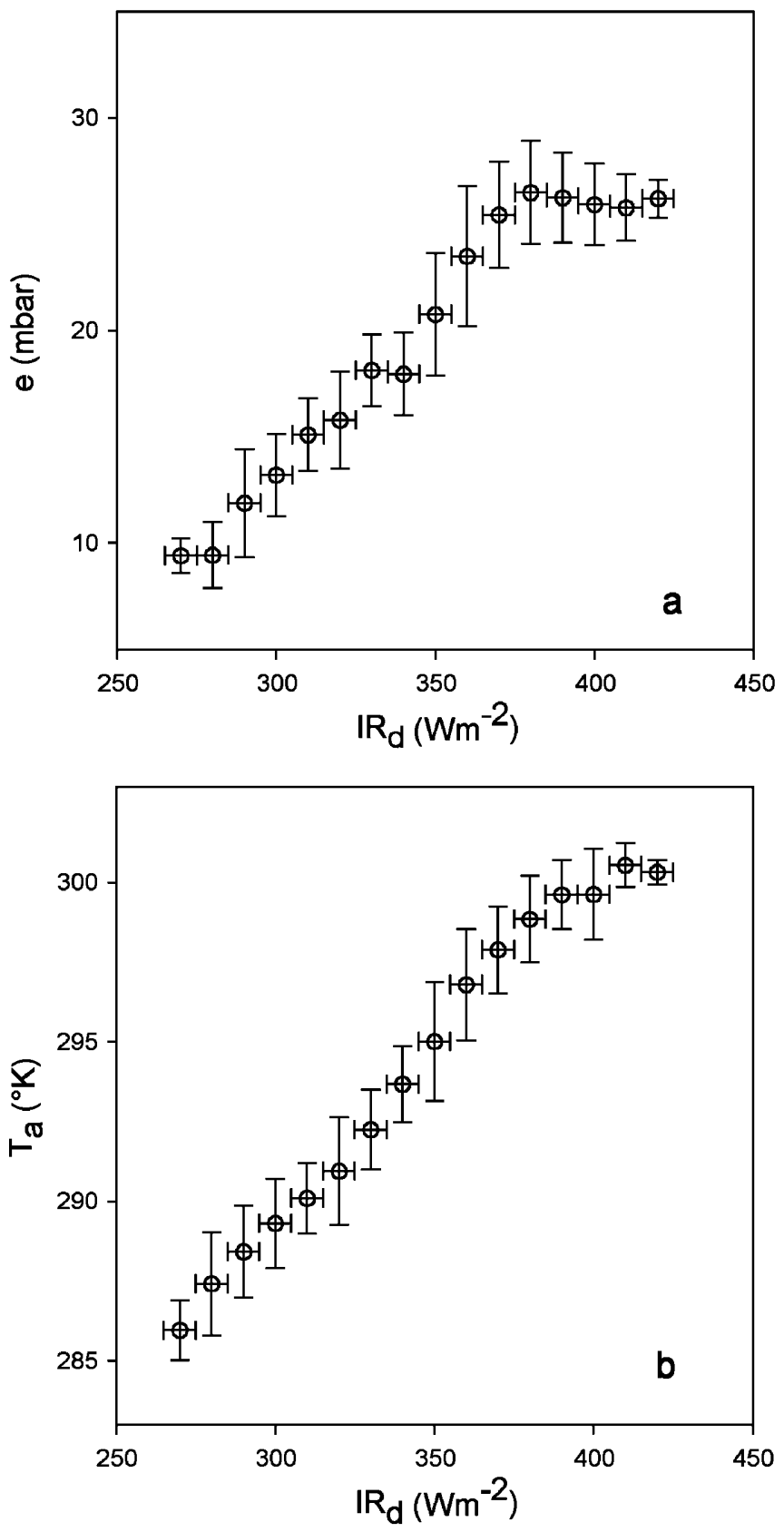

Fig. 1. a Surface water vapour tension and $\mathbf{b}$ surface air temperature versus clear-sky net longwave radiation. The data have been averaged within a range of $\pm 5 \mathrm{~W} \mathrm{~m}^{-2}$. Bars are the standard deviations

temperature. The difference is still large, but the obtained values are more reasonable, since they are included in the range of variability of these two parameters (Fig. 1a, b). This result suggests that the difference between the Med and the open ocean condition is due not only to the larger amount of vapour in the lower atmosphere, but also to the warmer air. In our opinion, the warmer air may be one of the possible causes of the systematic failure of the radiative bulk formulae in the Med.

Finally, we have assumed unchanged the experimental values of the atmospheric input parameters and we have derived the $T_{s}$ values, which satisfy Eq. (1). The 
estimated $T_{s}$ are about $3 \mathrm{~K}$ higher than the measured ones. This result indicates that we have to increase the upwelling component of the longwave radiation budget in order to counterbalance the greater amount of the downwelling longwave radiation estimated by the formula. However, this is not realistic over the Med, where the observed $T_{s}$ values are already high.

In conclusion, this inverse analysis suggests that the failure of the formula by Clark et al. (1974) over the Med is due to the large values of both surface air temperature and surface water vapour tension.

Of course, the incident radiation on the sea surface is strongly affected by the whole atmospheric column. In the bulk formulae, all processes occurring during the passage of the radiation through the atmosphere are represented by very simple terms, where the surface values of few parameters are the only required variables. This implies the assumption that the values at the surface are mainly determined by the conditions of the overlying atmosphere rather than by the interactions with the surface.

The validity of Eq. (1) over a mid-latitude open ocean and the results of our analysis imply that the surface parameters over the open ocean are lower than those over the Med under the same amount of net longwave radiation. The Med larger values may be due to the warm sea surface, which modifies the atmosphere near the interface by warming the air and increasing the evaporation.

\section{Peculiarities of climate regime}

In the bulk formulae the seasonal variability of the surface meteorological parameters is assumed to be sufficient to reproduce the seasonal variability of the radiative fluxes. Schiano (1996), by analysing Reed's (1977) formula for the solar radiation, has shown different results. She found that the overestimation of the clear sky insolation is larger during the summer. In order to adjust the algorithm, she chose the water vapour density as a climatic index to define the warm and cold seasons over the Med. Here, we try to carry on the same analysis on the longwave radiation over the Med. The test is performed by comparing direct measurements of net longwave radiation with predictions obtained by the formula of Clark et al. (1974).

The measurement-prediction ratios are examined according to the water vapour density $(\rho)$ (Fig. 2). This result reveals that the ratio between measurement and prediction of $I R_{n}$ is not constant, but presents a salient discontinuity for $\rho$ of about $16 \mathrm{~g} \mathrm{~m}^{-3}$. Values larger than $16 \mathrm{~g} \mathrm{~m}^{-3}$ denote warm and wet air masses, which can be found over the Med from May to October.

Therefore, this value can be assumed as a "threshold". Since the water vapour density may be lower than $16 \mathrm{~g} \mathrm{~m}^{-3}$ in the summer months, this threshold does not define winter from summer, but only evidences warm and wet weather condition.

In order to investigate the discontinuity, the $I R_{n}$ data are analysed according to $\rho$ (Fig. 3). The figure shows an

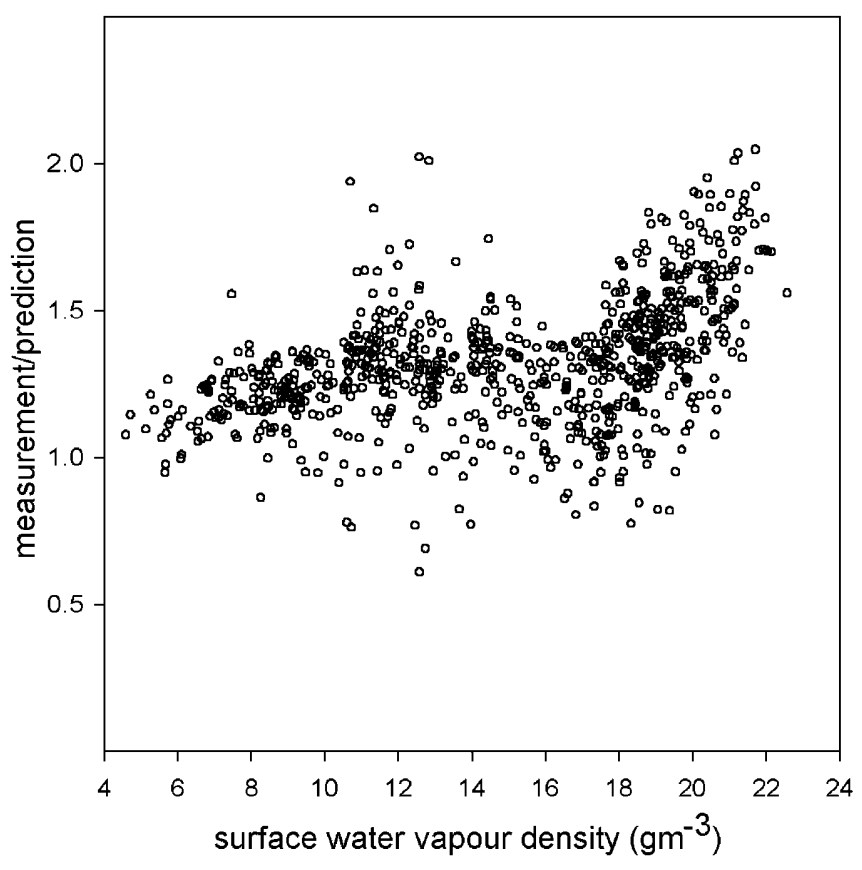

Fig. 2. Ratio between hourly measurements of clear-sky net longwave radiation and predictions using the formula of Clark et al. (1974) versus surface water vapour density

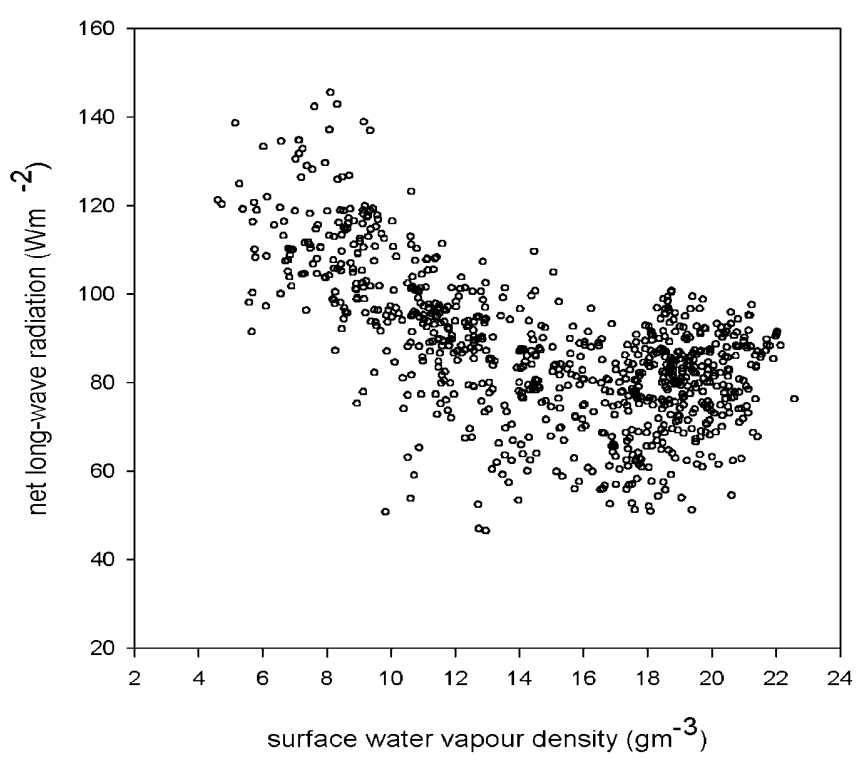

Fig. 3. Hourly measurements of net longwave radiation versus surface water vapour density

elbow-like pattern with a slope change occurring at the threshold value of the water vapour density. Below this value, the $I R_{n}$ decreases with the increase of $\rho$, while the trend is reversed over the threshold, (on the contrary, the estimates obtained by the formula of Clark et al. (1974) always show a decreasing trend). The discontinuity observed in Fig. 2 is due to this different behaviour. This result indicates that a single monotonic equation is not suitable for representing the net longwave radiation all year round. 
At the present time, the only net longwave radiation bulk formula developed for the Med is the formula of Bignami et al. (1995). We have tried to revise it by analysing its version for clear-sky, which is expressed as:

$I R_{n}=\varepsilon \sigma T_{s}^{4}-\sigma T_{a}^{4}(0.653+0.0053 e)$

Our analysis evidences that the ratio between the measured $I R_{n}$ and the estimate by Eq. (2) still shows a discontinuity (Fig. 4), even with the predictions close to the measurements.

According our opinion, the discontinuity is due to the function used by Bignami et al. (1995) for representing the atmospheric emissivity $\varepsilon_{a}$ :

$\varepsilon_{\mathrm{a}}=I R_{d} / \sigma T_{a}^{4}=(0.653+0.0053 e)$

which is a monotonic function of the surface water vapour tension.

The $I R_{d} / \sigma T_{a}^{4}$ ratio, shown in Fig. 5, shows, that this approximation is not correct in all cases. The relationship between the atmospheric emissivity and the amount of surface water vapour is nearly linear over a limited range of values. When wet and warm air conditions prevail, the previous relationship is not adequate. The increase of the water vapour tension does not correspond always to an increase of the atmospheric emissivity, i.e. under particular conditions the variation of the $I R_{d}$ is not related to the variation of surface water vapour.

The breakdown of this relationship indicates a large variation of the atmospheric features. We can state that the atmospheric thermal radiation and, consequently, the $I R_{n}$ do not simply follow seasonal cycles, but have patterns that are more complicated.

The formula of Bignami et al. (1995) has been defined by using a data set in which few measurements were collected under warm and wet condition. The

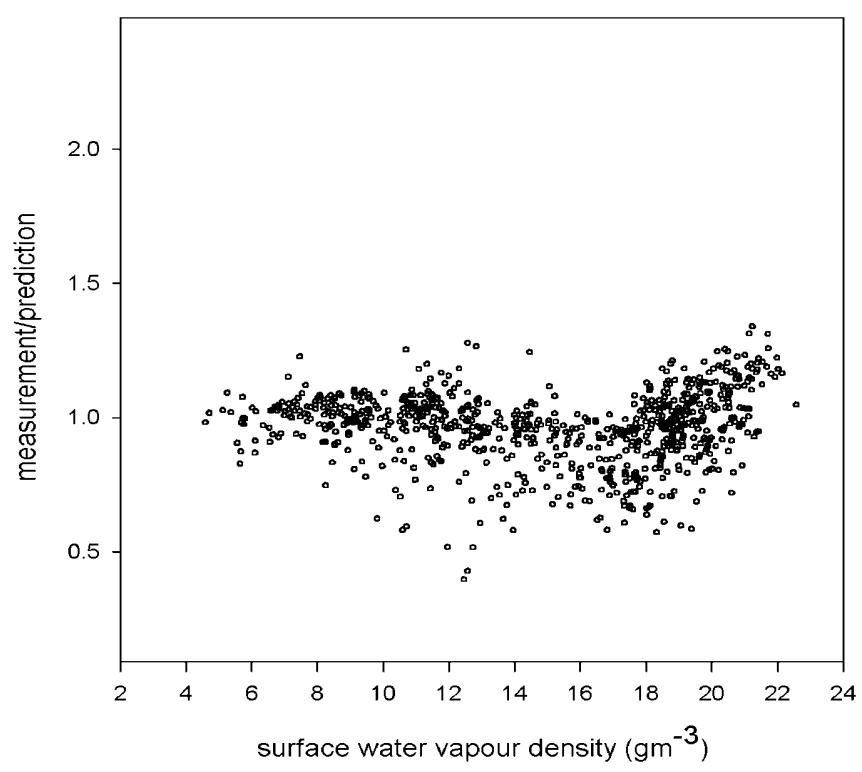

Fig. 4. Ratio between hourly measurements of clear-sky net longwave radiation and predictions by the formula of Bignami et al. (1995) versus surface water vapour density

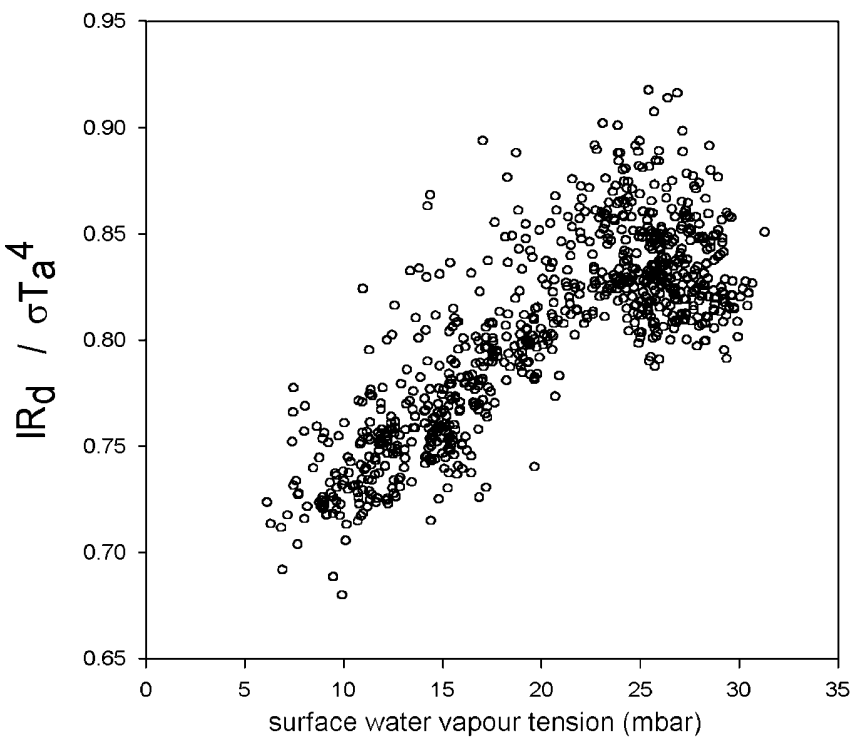

Fig. 5. $I R_{d} / T_{a}^{4}$ versus surface water tension

scarcity of these observations did not allow us to detect the discontinuous behaviour of the longwave radiation and to adequately resolve it.

We note that the pattern in Fig. 3 is very similar to those found in studies on the relationships between the outgoing longwave radiation (OLR) and the $T_{s}$. Previous analyses have shown that the relationship between the OLR and the $T_{s}$ changes the sign of its slope from mid-latitude areas to tropical zone (Raval et al., 1994; Lau et al., 1997). The well-known drop in OLR with increasing $T_{s}$ occurs in the tropics and is correlated to the increase of the amount of water vapour in the troposphere (Raval et al., 1994). In other words, over the open ocean the relationships between the amount of OLR and the surface meteorological parameters vary with the latitude.

Since the OLR and the $I R_{n}$ are strongly related, it is reasonable to assume that their behaviours have similar dependence on the climate regime. Then, we can state that the discontinuities in Figs. 3 and 5 are due to the peculiarities of the Med climate (in particular the WMed). In fact, it is known that the WMed shows a well-marked seasonal variation. During the cold season, a strong cyclonic activity is present and clear-sky conditions occur only when dry and cold air masses move from the north into the WMed. Over the warm sea, these air masses are rapidly warmed from below and collect moisture, but they remain appreciably colder and drier than the tropical air. In general, the $T_{s}$ increases more slowly than the air temperature. During the summer, the subtropical high-pressure activity shifts into the basin and stabilizes the weather conditions. When this takes place, the warm and wet air masses prevail and the surface water vapour tension is close to the saturated value. The warming due to the strong insolation makes the $T_{s}$ and the $T_{a}$ values almost the same at the air-sea interface (Meteorological Office, 1962). Clear-sky condition predominates, but the 
atmospheric turbidity is increased and a haze layer due to the Sahara dust is quite common in the upper atmosphere (Reiter, 1975). Under clear-sky, when warm and moist conditions prevail, the tropical characteristics of the Med overcome the mid-latitude features, even if these features are always altered as revealed by the high surface values of $T_{a}$ and water vapour tension during the cold months. Thus, over Med the relationships between the amount of downward radiation and the surface meteorological parameters vary over time, because the seasonal differences are very strong and their effects may be compared to a change of climatic regime. This suggests that different bulk formulae have to be developed according to the climate situation.

\section{Conclusions}

Previous studies have shown that the most-used radiative bulk formulae give estimates over the Med which systematically disagree with observed radiative fluxes.

Here, an investigation on this systematic discrepancy has been performed to show some peculiarities of the Med. The approach started from the assumption that the reliability of these bulk formulae depends on the climatic features of the area where they are applied. Formulae, which have been successfully tested over the open ocean, have been used over the Med, and their failure has been ascribed to some climatic features of the Med marine environment.

The analysis has been conducted only for clear-sky conditions by using direct radiative measurements collected during 13 cruises in the WMed in the last years.

The results indicate that the values of air temperature and water vapour tension at the sea surface of the Med are much higher than those measured over a midlatitude open ocean under the same amount of incident radiation. This may be due to the warm sea surface, which interacts with the overlying atmosphere and strongly modifies the weather parameters at the interface.

The Med presents a "twofold climate regime", i.e. both tropical and mid-latitude aspects are playing a role. This complex climate system is shown by the behaviour of the bulk formulae, making the disagreement between them and the observations not constant. The empirical relationship between radiative fluxes and surface parameters varies according to the climate regime.

Until now, the bulk formulae have been adjusted for the Med through simple modifications of the numerical coefficients. The present study shows that this is not a correct procedure. Future work will be devoted to develop suitable bulk formulae for the Med, taking into account the impact of different climate situations.

Acknowledgements. We would like to acknowledge the assistance of the captains and the crews of $\mathrm{R} / \mathrm{V}$ Minerva and $\mathrm{R} / \mathrm{V}$ Urania. The European Community under contract MAS3-CT96-0051 MTP II MATER founded the present work.

Topical Editor N. Pinardi thanks S. Josey and another referee for this help in evaluating this paper.

\section{References}

Bignami, F., S. Marullo, R. Santoleri, and M. E. Schiano, Long wave radiation budget in the Mediterranean Sea, J. Geophys. Res., 100(C2), 2501-2514, 1995.

Clark, N. E., L. Eber, R. M. Laurs, J. A. Renner, and J. F. T. Saur, Heat exchange between ocean and atmosphere in the eastern North Pacific for 1961, 71. NOAA Tech. Rep. NMSF SSRF682, US Department of Commerce, Washington D.C., 1974.

Fairall, C. W., P. O. G. Persson, E. F. Bradley, R. E. Payne, and S. P. Anderson, A new look at calibration and use of Eppley precision infrared radiometers. Part I: theory and application, J. Atmos. Ocean. Technol., 15, 1229-1242, 1998.

Katsaros, K. B., Parameterization schemes and models for estimating the surface radiation budget, in Surface waves and fluxes, vol II, remote sensing, Eds. Geernaert, G. L. and W. J. Plant, 339-368, Kluwer, Norwell, Mass., 1990.

Gilman, C., and C. Garrett, Heat flux parameterizations for the Mediterranean Sea: the role of atmospheric aerosols and constraints from the water budget, J. Geophys. Res., 99(C3), 5119-5134, 1994.

Hansterath, S., and P. J. Lamb, Heat budget atlas of the tropical Atlantic and Eastern Pacific Oceans, 90 pp. University of Wisconsin Press, Madison, 1978.

Josey, S., D. Oakley, and R. W. Pascal, On estimating the atmospheric longwave flux at the ocean surface from ship meteorological reports, J. Geophys. Res., 102(C13), 27 96127 972, 1997.

Lau, K. M., H. T. Wu, and S. Bony, The role of large-scale atmospheric circulation in the relationship between tropical convection and sea surface temperature, J. Clim., 10, 381-392, 1997.

Meteorological Office, Weather in the Mediterranean, 2nd edn., 362 pp., Her Majesty's Stationery Office, London, 1962.

Raval, A., A. H. Oort, and V. Ramaswamy, Observed dependence of outgoing longwave radiation on sea surface temperature and moisture, J. Clim., 7, 807-821, 1994.

Reiter, E. R., Weather phenomena of the Mediterranean basin, Part 1, Environmental Prediction Research Facility, Naval Postgraduate School, Monterey, California 93940, 1975.

Reed, R. K., On estimating insolation over the ocean, J. Phys. Oceanogr., 7, 482-485, 1977.

Schiano, M. E., R. Santoleri, F. Bignami, and R. M. Leonardi, Airsea interaction measurements in the western Mediterranean Sea during the Tyrrhenian Eddy Multi-Platform Observations Experiment, J. Geophys. Res., 98(C2), 2461-2474, 1993.

Schiano, M. E., Insolation over the western Mediterranean Sea: a comparison of direct measurements and Reed's formula, J. Geophys. Res., 101(C2), 3831-3838, 1996. 